\title{
Opposite magnetic polarity of two photospheric lines in single spectrum of the quiet Sun
}

\author{
R. Rezaei, R. Schlichenmaier, W. Schmidt, and O. Steiner
}

\begin{abstract}
Kiepenheuer-Institut für Sonnenphysik, Schöneckstr. 6, 79104 Freiburg, Germany
e-mail: [rrezaei; schliche;wolfgang; steiner] akis . uni-freiburg.de
\end{abstract}

Received 8 March 2007 / Accepted 24 April 2007

\section{ABSTRACT}

\begin{abstract}
Aims. We study the structure of the photospheric magnetic field of the quiet Sun by investigating weak spectro-polarimetric signals. Methods. We took a sequence of Stokes spectra of the Fe I $630.15 \mathrm{~nm}$ and $630.25 \mathrm{~nm}$ lines in a region of quiet Sun near the disk center, using the POLIS spectro-polarimeter at the German VTT on Tenerife. The line cores of these two lines form at different heights in the atmosphere. The $3 \sigma$ noise level of the data is about $1.8 \times 10^{-3} I_{\mathrm{c}}$.

Results. We present co-temporal and co-spatial Stokes- $V$ profiles of the Fe I $630 \mathrm{~nm}$ line pair, where the two lines show opposite polarities in a single spectrum. We compute synthetic line profiles and reproduce these spectra with a two-component model atmosphere: a non-magnetic component and a magnetic component. The magnetic component consists of two magnetic layers with opposite polarity: the upper one moves upwards while the lower one moves downward. In-between, there is a region of enhanced temperature. Conclusions. The Stokes- $V$ line pair of opposite polarity in a single spectrum can be understood as a magnetic reconnection event in the solar photosphere. We demonstrate that such a scenario is realistic, but the solution may not be unique.
\end{abstract}

Key words. Sun: photosphere - Sun: magnetic fields

\section{Introduction}

Stokes polarimetry provides detailed information about the magnetic field and its interaction with the plasma in the solar photosphere and chromosphere (Stenflo 1994; Landi degl'Innocenti \& Landolfi 2004). While most of the observed Stokes- $V$ profiles in active regions and the network are close to antisymmetric with a low degree of asymmetry, abnormal and strongly asymmetric $V$ profiles are common in the inter-network (Sigwarth 2001; Lites 2002). The classification of Sigwarth et al. (1999) presents abnormal $V$ profiles with a single lobe, two lobes with identical polarities, and $Q$-like, or pathological profiles with four or more lobes. There are indications from observations and 3D simulations that the degree of asymmetry and the fraction of abnormal $V$ profiles increase with decreasing magnetic flux (Sigwarth et al. 1999; Khomenko et al. 2005). While most of the pathological profiles can be reconstructed with models consisting of two or more magnetic components, Grossmann-Doerth et al. (2000) and Steiner (2000) have shown that one-component models can also account for a large variety of $V$ profiles provided that magnetic, velocity, and temperature gradients are large enough.

An opposite polarity (OP) Stokes- $V$ profile is a set of two $V$ profiles of two different spectral lines, recorded in a strictly co-temporal and co-spatial observation, that shows different polarities. Sánchez Almeida et al. (2003) report opposite polarity $V$ profiles between the visible $(630 \mathrm{~nm})$ and the infrared $(1.56 \mu \mathrm{m})$ neutral iron lines observed at the French-Italian solar telescope THÉMIS and the German Vacuum Tower Telescope (VTT), respectively. This work was questioned later by Khomenko et al. (2005), who showed that different seeing conditions for the two data sets can spuriously produce OP profiles.
In this paper, we present observations of a quiet Sun region close to the disk center with the POlarimetric Littrow Spectrograph (POLIS, Schmidt et al. 2003; Beck et al. 2005a) that show a few OP Stokes- $V$ profiles. Each set of OP profiles consists of the two iron lines at $\lambda \lambda 630.15$ and $630.25 \mathrm{~nm}$ that are part of a single spectrum recorded strictly simultaneously. The formation heights of these two lines span different layers in the photosphere (Cabrera Solana et al. 2005; Khomenko \& Collados 2007, and references therein). We study one set of these profiles in detail and argue that it hints at a magnetic reconnection event in the solar photosphere.

\section{Observations and data reduction}

A sequence of spectra taken in a quiet Sun region close to the disk center $(\cos \theta=0.99)$, was observed with the VTT in Tenerife, July 07, 2006. The seeing was good and stable during the observation. The Kiepenheuer Adaptive Optics System was used for maximum spatial resolution and image stability (von der Lühe et al. 2003). Each of the 38 scans consists of 16 slit positions. The scanning step size and spatial sampling along the slit were 0.48 and 0.29 arcsec, respectively. The scanning cadence was about $97 \mathrm{~s}$.

Full Stokes profiles of the neutral iron lines at 630.15 and $630.25 \mathrm{~nm}$ and the Stokes- $I$ profile of the Ca II H line were observed strictly simultaneously with the red $(630 \mathrm{~nm})$ and blue (396.8 nm) channels of POLIS. The average continuum contrast (rms) of the POLIS Stokes- $I$ maps is $\approx 3.4 \%$ of $I_{\mathrm{c}}$. An absolute velocity calibration was performed using the telluric $\mathrm{O}_{2}$ line at $630.20 \mathrm{~nm}$ (Rezaei et al. 2006). The spectro-polarimetric data of the red channel were corrected for instrumental effects and telescope polarization with the procedures described by 

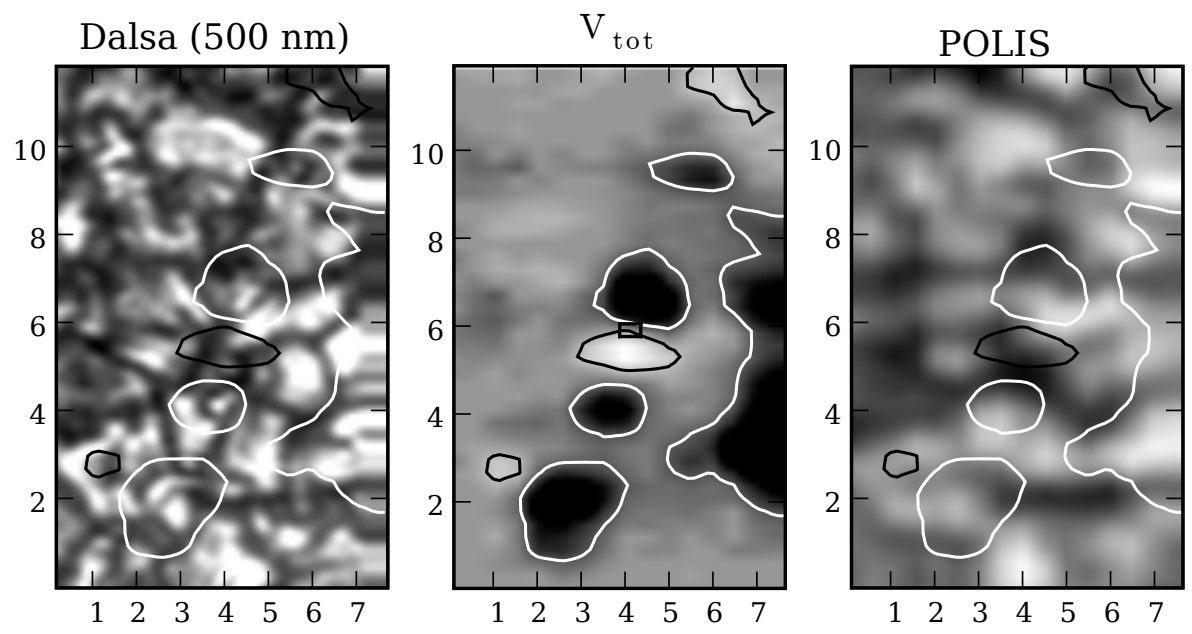

Fig. 1. Co-spatial and co-temporal data of the speckle reconstructed continuum image at $500 \mathrm{~nm}$ (left), $V_{\text {tot }}$ map (middle), and Stokes-I continuum map (right). Contours of the $V_{\text {tot }}$ map are overplotted on all panels. The position of the opposite polarity profile is marked with a rectangle, which also shows the POLIS pixel size. The horizontal is the scan direction and the vertical is the slit direction. In the $V_{\text {tot }}$ map, white and black indicate negative and positive polarities, respectively (for definition of positive/negative polarity, see Fig. 2). Tickmarks indicate arcsec.

Beck et al. (2005a,b). The rms noise level of the Stokes parameters in the continuum is $\sigma=6.0 \times 10^{-4} I_{\mathrm{c}}$.

Simultaneously, a continuum speckle channel in POLIS recorded a larger field of view at $500 \mathrm{~nm}$. The speckle reconstruction was performed using the Kiepenheuer-Institut Speckle Imaging Package (Mikurda \& von der Lühe 2006; Wöger 2006). The spatial resolution of the reconstructed image is about 0.3 arcsec (cf. Fig. 1, left panel). We used the POLIS intensity map and the reconstructed image to align the data.

We define the signed $V_{\text {tot }}$ as follows:

$V_{\text {tot }}=\frac{\int_{\lambda_{\mathrm{b}}}^{\lambda_{0}} V(\lambda) \mathrm{d} \lambda-\int_{\lambda_{0}}^{\lambda_{\mathrm{r}}} V(\lambda) \mathrm{d} \lambda}{I_{\mathrm{c}}\left(\lambda_{\mathrm{r}}-\lambda_{\mathrm{b}}\right)}$,

where $\lambda_{0}$ is the zero-crossing wavelength of the Stokes- $V$ profile and $\lambda_{\mathrm{r}}$ and $\lambda_{\mathrm{b}}$ denote fixed wavelengths in the red and blue continuum of the lines (Lites et al. 1999). The continuum speckle image and the POLIS Stokes- $I$ map are shown in the left and right panels of Fig. 1, respectively. The middle panel of Fig. 1 shows the $V_{\text {tot }}$ map in which the position of the OP profile is marked with a rectangle that also shows the POLIS pixel size. It is located between two patches of opposite polarities. The OP profile (exposure time $5 \mathrm{~s}$ ) was recorded within the time window of $15 \mathrm{~s}$ used for the speckle burst (left panel, Fig. 1). This image and the continuum map (right panel, Fig. 1) indicate that the lower patch was co-spatial with an intergranular vertex (white patch in the $V_{\text {tot }}$ map). Therefore, the $V$ profiles in the lower patch show a redshift, which is also the case for the $630.25 \mathrm{~nm}$ OP profile.

Figure 2 shows the OP profile. The positive (negative) magnetic polarity in this figure corresponds to black (white) in the $V_{\text {tot }}$ map. Both lines have positive amplitude and area asymmetries. The $630.15 \mathrm{~nm}$ line of the OP profile has a blueshifted zero-crossing of $v_{\mathrm{zc}} \approx-1 \mathrm{~km} \mathrm{~s}^{-1}$, while the $630.25 \mathrm{~nm}$ line has a redshifted zero-crossing of $v_{\mathrm{zc}} \approx+2 \mathrm{~km} \mathrm{~s}^{-1}$.

The OP profile along with its neighboring profiles are shown in Fig. 3. In this figure, the slit direction is vertical and the OP profile is at the center. The Stokes- $V$ profiles above and below the OP profile show strong $V$ signals of normal shape which shows that the OP profile was located at the center of a polarity reversal. The polarity of the $630.25 \mathrm{~nm}$ line of the OP profile is the same as that of the lower pixels and the polarity of the $630.15 \mathrm{~nm}$ line is identical to that of the upper ones. The $V$ profile left to the OP profile (Fig. 3, second row, left) is also strange: a pathological profile for the $630.15 \mathrm{~nm}$ line and a regular profile of the $630.25 \mathrm{~nm}$ line with a polarity similar to the $630.25 \mathrm{~nm}$

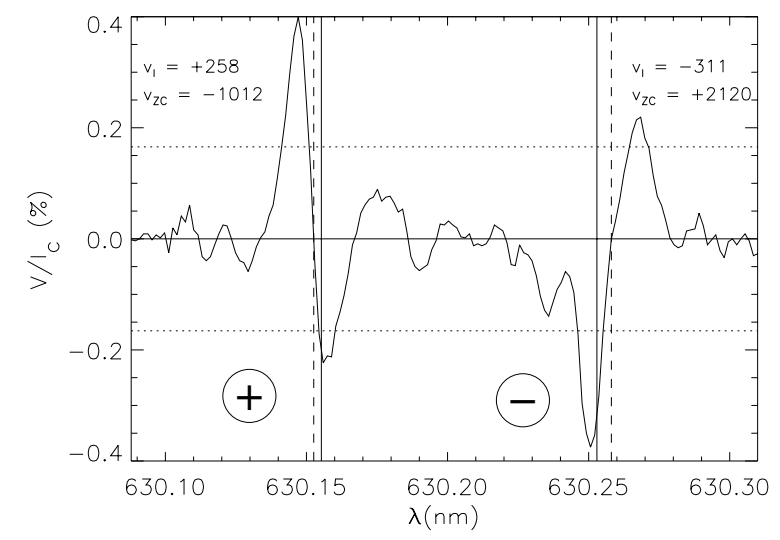

Fig. 2. Stokes- $V$ line pair that shows opposite polarity in the 630.15 and $630.25 \mathrm{~nm}$ lines. The position of the Stokes- $I$ line-core and the Stokes- $V$ zero-crossing are indicated by vertical solid and dashed lines, respectively. The line-core $\left(v_{\mathrm{I}}\right)$ and zero-crossing $\left(v_{\mathrm{zc}}\right)$ velocities are in $\mathrm{m} \mathrm{s}^{-1}$.

line in the OP profile. The same is true for one scan-step after the OP profile (Fig. 3, second row, right column): a normal $V$ profile at $630.15 \mathrm{~nm}$ with a polarity identical to the $630.15 \mathrm{~nm}$ line of the OP profile and no signal for the $630.25 \mathrm{~nm}$ line.

Note that the OP profile can not be reproduced by seeing effects as the adjacent profiles above and below the central OP profile in Fig. 3 have incompatible shifts, line widths, and amplitudes: a least-squares fit to a superposition of the adjacent upper and lower profiles yields residuals exceeding $2 \sigma$. Independently, we measure from the speckle burst that the standard deviation of the image motion during the exposure was only about 0.1 arcsec. Therefore the OP profile is not due to seeing effects.

\section{The model profile}

The basic properties of the observed OP profile are: (i) the opposite polarities of the $V$ profiles at $630.15 \mathrm{~nm}$ and $630.25 \mathrm{~nm}$, (ii) the velocity of the deeper forming line $(630.25 \mathrm{~nm})$ is positive (downward) and the velocity of the higher forming line $(630.15 \mathrm{~nm})$ is negative (upward); and (iii) both lines show positive area and amplitude asymmetries. The vicinity of the OP profile is characterized by two patches of opposite polarity that are separated by only one resolution element as indicated by the rectangle in the middle panel of Fig. 1 and in the profile array of Fig. 3. This configuration suggests that we witness an electric current sheet in the intermediate atmospheric layers with 

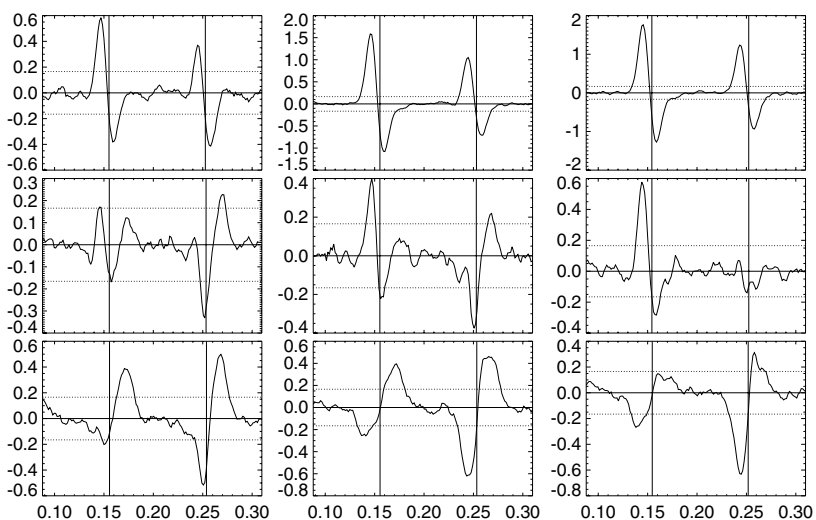

Fig. 3. The OP Stokes- $V$ profile (center) and its surrounding profiles. Abscissae show the wavelength $(\lambda-630 \mathrm{~nm})$. The slit direction is vertical. Other parameters are like in Fig. 2.

magnetic field of one polarity in deeper layers and of the opposite polarity in higher layers. The field lines should be inclined with respect to the line-of-sight since there is a $U$ signal in the observed Stokes parameters (Fig. 5). The finding of upward velocity in the higher layer and downward velocity in the deeper layer presents evidence for magnetic reconnection, since such an event produces a bipolar jet. In the central region in-between the two magnetic polarities one expects an enhanced temperature due to Joules heating (gray scale, Fig. 4). This basic picture is sketched in the left panel of Fig. 4. Based on these ideas, we construct in the following a quantitative model which allows us to compute a set of synthetic Stokes profiles for a direct comparison with the observed OP profile.

We use the SIR code (Ruiz Cobo \& del Toro Iniesta 1992) to synthesize the Stokes profiles of the Fe I $630 \mathrm{~nm}$ pair. In order to fit the degree of polarization and to account for straylight, the model atmosphere must contain a field free (non-magnetic) component beside the magnetic component. The non-magnetic component is taken to be the HSRA model atmosphere (Gingerich et al. 1971). This thermal stratification is also used for the magnetic component with a slight modification: in an intermediate layer around $\log \tau_{500 \mathrm{~nm}} \approx-1.2$, we introduce a temperature bulge. At the peak of this hot bulge, it is $270 \mathrm{~K}$ hotter than in the HSRA atmosphere. The hot bulge is partly field free and partly overlaps with the magnetic field of the deeper layer. Above and below this hot bulge, the velocity and magnetic field have opposite orientations. The ranges of non-vanishing magnetic field strength and velocity overlap, but do not have their peak values at the same optical depth. Constant values for the inclination of the magnetic field with respect to the vertical $\left(35^{\circ}\right)$ and the azimuth $\left(135^{\circ}\right)$ are used to fit the ratios between $Q, U$, and $V$. The model atmosphere that was finally used to reproduce the OP profile is shown in Fig. 4 (right). All structure elements of this model atmosphere are necessary ingredients for a successful reproduction of the OP profile. In particular, the temperature bump is essential to reproduce the different V-profile polarities when using only one line-of-sight along which two magnetic polarities are present. This demonstrates that OP profiles can be synthesized with a realistic model, but note that this solution may not be unique.

To obtain the filling factor of the magnetic atmosphere, we perform an inversion of the regular Stokes profiles in the surrounding pixels of the OP profile. The inversion set up is similar to that of Bellot Rubio \& Beck (2005), except that we assume a constant value for the stray light and allow for a linear gradient
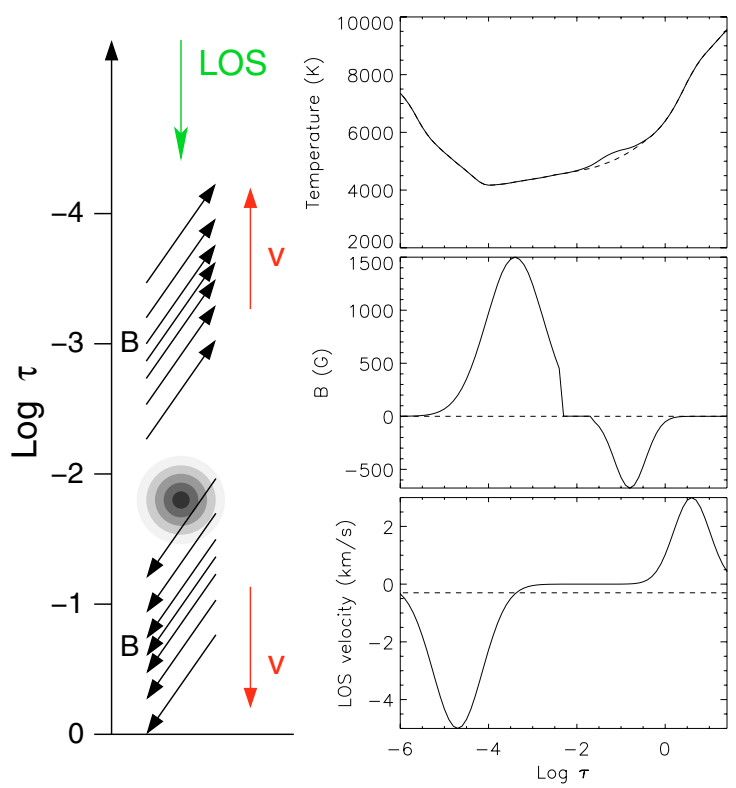

Fig. 4. Left: schematic configuration of the magnetic field, the line-ofsight velocity, and the temperature enhancement (gray scale). Right: stratification of the atmosphere as a function of optical depth for the magnetic (solid) and non-magnetic (dashed) components. From top to bottom: temperature, magnetic field strength (with the the sign denoting the polarity), and line-of-sight velocity. The non-magnetic atmosphere has a convective blueshift of $300 \mathrm{~m} \mathrm{~s}^{-1}$.

of the line-of-sight velocity in the magnetic component. From these inversions we obtain magnetic filling factors of $10-30 \%$, so we assume a filling factor of $20 \%$ for our model atmosphere.

The upper layer of magnetic field along with the bump of negative velocity leads to a very asymmetric $V$ profile for both lines. This profile has the polarity of the $630.15 \mathrm{~nm}$ line of the OP profile and shows a higher amplitude in $630.15 \mathrm{~nm}$ than in $630.25 \mathrm{~nm}$. The lower-layer magnetic field of opposite polarity produces an almost antisymmetric $V$ profile at $630.25 \mathrm{~nm}$, but the profile at $630.15 \mathrm{~nm}$ is asymmetric. The combination of these two components gives the final fit to the data that is shown in Fig. 5. The quality of the fit is satisfactory considering that we only used one single line-of-sight for this complex topology. With a slight shift of the velocity peaks we are also able to reproduce the pathological profile to the left to the OP profile (Fig. 3).

\section{Discussion}

The magnetic area with polarity rendered in dark in the $V_{\text {tot }}$ map of Fig. 1 was present throughout the observing time of 64 min and was persistently bright in $\mathrm{Ca}$ II $\mathrm{H}$, which suggests that it belonged to network magnetic fields. On the contrary, the opposite polarity, white patch, visible in the center of the $V_{\text {tot }}$ map, was a transient feature - it assembled from diffuse flux of white polarity, concentrated and intensified to the white patch seen in Fig. 1, before it rapidly weakened. Within the scanning cadence of $97 \mathrm{~s}$ it virtually disappeared, not without influencing the opposite polarity (black) neighboring patches to the upper and lower sides, which weakened during this time. Such events must frequently take place considering that the magnetic flux in the quiet network is permanently replaced by and in interaction with mixed polarity flux (Schrijver et al. 1997). When flux concentrations of opposite polarity collide, flux cancellation and magnetic field reconnection are likely to be involved (Zwaan 1987). At the reconnection site we expect conversion from magnetic to 

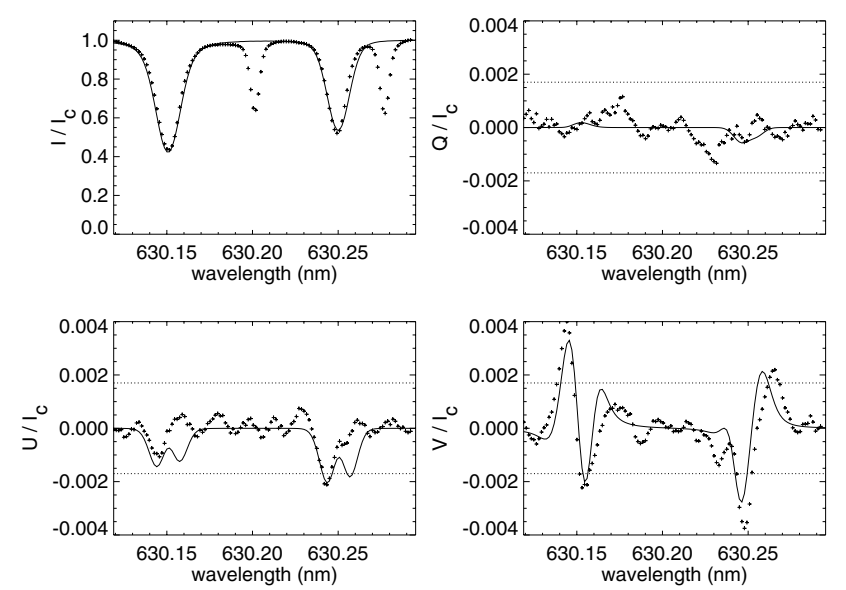

Fig. 5. Comparison between the observed Stokes profiles (pluses) and the synthesized ones (solid lines). The horizontal dotted lines show the $3 \sigma$ noise level.

thermal energy and the formation of a bipolar jet: processes that are compatible with the temperature bulge in between the two layers of oppositely directed velocities and magnetic fields of the atmosphere of Fig. 4 and with the observed flux cancellation and weakening.

For a check of compatibility with the thermal, kinetic, and magnetic energy fluxes required in a reconnection event, we first consider a control volume given by the size of one POLIS pixel of $A_{p}=\Delta_{x} \times \Delta_{y}=350 \mathrm{~km} \times 210 \mathrm{~km}$ and the height range spanned by the FWHM of the temperature bulge of $h=135 \mathrm{~km}$, which encompasses a mass of $m=1.3 \times 10^{12} \mathrm{~kg}$. With a bulge peak of $\delta T=270 \mathrm{~K}$ we obtain from Newton's law of cooling a radiative heat loss from this volume of

$\dot{Q}=16 \sigma \kappa T^{3} \delta T m f=9 \times 10^{16} \mathrm{~W}$,

where $\sigma$ is the Stefan-Boltzmann constant, $\kappa=1.1 \times$ $10^{-2} \mathrm{~m}^{2} \mathrm{~kg}^{-1}$ the opacity corresponding to the mean density of $1.3 \times 10^{-4} \mathrm{~kg} \mathrm{~m}^{-3}, T=5100 \mathrm{~K}$ the corresponding temperature of the unperturbed background atmosphere, and $f=0.2$ the filling factor. This radiative energy flux should be of the same order of magnitude as the kinetic energy flux,

$F_{\text {kin }}=(1 / 2) \rho v^{3} A_{p} f$

carried by the bipolar reconnection jets. Using the values of the lower, heavier (downward moving) layer with $v_{\text {LOS }}=3 \mathrm{~km} \mathrm{~s}^{-1}$ and $\rho=3.0 \times 10^{-4} \mathrm{~kg} \mathrm{~m}^{-3}$, and assuming the velocity to be directed parallel to the magnetic field we obtain $F_{\text {kin }}=1 \times 10^{17} \mathrm{~W}$. The kinetic energy of the upwards moving jet is negligible, because of the lower density. The kinetic and thermal energy fluxes need to be sustained by a corresponding influx of magnetic energy of

$F_{\text {mag }}=v\left(B^{2} / 2 \mu_{0}\right) \Delta_{x} h \sqrt{f}$.

With $B=0.1 \mathrm{~T}$ we obtain $F_{\text {mag }}=1 \times 10^{17} \mathrm{~W}$ if the influx velocity is $1.2 \mathrm{~km} \mathrm{~s}^{-1}$, which is a reasonable value for the horizontal velocity of photospheric magnetic flux concentrations.

Photospheric magnetic reconnection has previously been studied by Litvinenko (1999) and Takeuchi \& Shibata (2001). Since we observe a flux intensification prior to flux canceling for the white polarity patch of Fig. 1, the scenario of Takeuchi \& Shibata (2001) consisting of reconnection induced by convective intensification may apply to this event.
We would like to stress that the model presented here is not necessarily unique and that other model atmospheres may also reproduce the data. However, we expect any alternative model to show strong gradients in velocity and magnetic field strength.

\section{Conclusion}

We have discovered several sets of opposite polarity Stokes$V$ profiles. Each set is a single spectrum of the 630.15 and $630.25 \mathrm{~nm}$ neutral iron lines pertaining to a single resolution element. We use a model atmosphere consisting of a non-magnetic and one magnetic component for synthesizing the observed profiles. The magnetic component contains strong gradients along the line-of-sight in the magnetic field strength and the velocity. In particular, the higher and deeper layers have opposite magnetic polarity. In accordance with the observed red- and blue-shifts of the spectral lines, the proposed model atmosphere contains a bipolar flow of material along the line-of-sight and it contains a temperature enhancement in-between. These atmospheric elements provide evidence of magnetic reconnection in the solar photosphere. Our estimated values for the thermal, kinetic, and magnetic energy fluxes are also compatible with a reconnection event.

Acknowledgements. We wish to thank C. Beck and F. Wöger for their assistance during observation and data reduction. We are grateful to J. Bruls, O. von der Lühe, and E. Khomenko for their useful comments. The POLIS instrument has been a joint development of the High Altitude Observatory (Boulder, USA) and the Kiepenheuer-Institut. Part of this work was supported by the Deutsche Forschungsgemeinschaft (SCHM 1168/8-1).

\section{References}

Beck, C., Schlichenmaier, R., Collados, M., Bellot Rubio, L., \& Kentischer, T. 2005a, A\&A, 443, 1047

Beck, C., Schmidt, W., Kentischer, T., \& Elmore, D. 2005b, A\&A, 437, 1159 Bellot Rubio, L. R., \& Beck, C. 2005, ApJ, 626, L125

Cabrera Solana, D., Bellot Rubio, L. R., \& del Toro Iniesta, J. C. 2005, A\&A, 439,687

Gingerich, O., Noyes, R. W., Kalkofen, W., \& Cuny, Y. 1971, Sol. Phys., 18, 347 Grossmann-Doerth, U., Schüssler, M., Sigwarth, M., \& Steiner, O. 2000, A\&A, 357,351

Khomenko, E. V., \& Collados, M. 2007, ApJ, accepted

Khomenko, E. V., Shelyag, S., Solanki, S. K., \& Vögler, A. 2005, A\&A, 442, 1059

Landi degl'Innocenti, E., \& Landolfi, M. 2004, Polarization in Spectral Lines (Astrophysics and Space Science Library, Kluwer Academic Publishers)

Lites, B. W. 2002, ApJ, 573, 431

Lites, B. W., Rutten, R. J., \& Berger, T. E. 1999, ApJ, 517, 1013

Litvinenko, Y. E. 1999, ApJ, 515, 435

Mikurda, K., \& von der Lühe, O. 2006, Sol. Phys., 235, 31

Rezaei, R., Schlichenmaier, R., Beck, C. A. R., \& Bellot Rubio, L. R. 2006, A\&A, 454, 975

Ruiz Cobo, B., \& del Toro Iniesta, J. C. 1992, ApJ, 398, 375

Sánchez Almeida, J., Domínguez Cerdeña, I., \& Kneer, F. 2003, ApJ, 597, L177

Schmidt, W., Beck, C., Kentischer, T., Elmore, D., \& Lites, B. 2003, Astron. Nachr., 324, 300

Schrijver, C. J., Title, A. M., van Ballegooijen, A. A., Hagenaar, H. J., \& Shine, R. A. 1997, ApJ, 487, 424

Sigwarth, M. 2001, ApJ, 563, 1031

Sigwarth, M., Balasubramaniam, K. S., Knölker, M., \& Schmidt, W. 1999, A\&A, 349,941

Steiner, O. 2000, Sol. Phys., 196, 245

Stenflo, J. O. 1994, Solar Magnetic Fields: Polarized Radiation Diagnostics (Astrophysics and Space Science Library, Kluwer Academic Publishers)

Takeuchi, A., \& Shibata, K. 2001, ApJ, 546, L73

von der Lühe, O., Soltau, D., Berkefeld, T., \& Schelenz, T. 2003, in Innovative Telescopes and Instrumentation for Solar Astrophysics, ed. S. L. Keil, \& S. V. Avakyan, Proc. SPIE, 4853, 187

Wöger, F. 2006, Ph.D. Thesis, Freiburg University

Zwaan, C. 1987, ARA\&A, 25, 83 